\title{
Solid Nucleation Mode Engine Exhaust Particles Detection at High Temperatures with an Advanced Half Mini DMA
}

\author{
Penelope Baltzopoulou, Anastasios D. Melas, Nickolas Vlachos, Danis Deloglou, \\ Eleni Papaioannou, and Athanasios G. Konstandopoulos
}

CERTH/CPERI

\begin{abstract}
Diesel and gasoline direct injection engines emit nucleation mode particles either under special conditions or as part of their normally emitted size distribution, respectively. Currently, European legislation excludes nucleation mode particles as particle number vehicle emission measurements are limited down to $23 \mathrm{~nm}$. The rationale behind such a cut-off size is based on the avoidance of significant uncertainties inherent in the sampling and measuring of sub-23 nm solid particles. However, the sub- $23 \mathrm{~nm}$ particles have drawn increased attention since a large fraction of particles emitted by modern vehicles lies in this size range. In this study we investigate the possibility of accurate nucleation mode particles detection by using the Advanced Half Mini Differential Mobility Analyzer (HMDMA). The Advanced HM-DMA system is able to classify aerosol particles in the mobility size range $5-30 \mathrm{~nm}$ with high resolution and fast spectrum acquisition that can accommodate a sample flow maintained at up to $200^{\circ} \mathrm{C}$. The unique ability to classify particles at high temperatures permits engine exhaust measurements without the need for aerosol conditioning. Initially, the Advanced HM-DMA hot operation mode accuracy is tested with reference aerosols against State-of-the-Art instruments. Thereafter, the Advanced HM-DMA is employed for measuring nucleation mode particles generated by a diesel engine using a single hot dilution step. Advanced HM-DMA measurements are compared to measurements performed with PMP protocol-compliant volatile particle removal system. The excellent agreement between the two measurements confirms the reliability of the Advanced HM-DMA hot operation mode and indicates the possibility of using a simplified conditioning setup for solid nucleation-mode particles measurement.
\end{abstract}

\section{Introduction}

Starting from the EURO 5b/6 standard, European legislation imposes a number limit for solid particles emitted by light-duty vehicles. The particle number limit was adopted, in addition to the more traditional mass-based measurements, in order to account for the health effects of emitted ultrafine particles of mobility diameter, $\mathrm{D}_{\mathrm{m}}<100 \mathrm{~nm}$ [1]. Such small exhaust particles do not contribute significantly to emissions in total mass but do contribute strongly to emissions in number. Solid particle number measurements for vehicle homologation are performed according to a protocol prepared by the Particle Measurement Programme (PMP) group [2] in which a cut-off size at $23 \mathrm{~nm}$ is currently implemented.

Page 1 of 8
According to the PMP protocol, the vehicle's raw exhaust is driven to a full flow dilution tunnel utilising a constant volume sampling (CVS) system. Possible volatile and semi-volatile material that condenses on solid particles or creates a separate nucleation mode is evaporated in a Volatile Particle Remover (VPR) and then particle number concentration is measured by a particle counter with a nominal cut-off size at $23 \mathrm{~nm}$. The VPR consists of two dilution stages: a first stage using hot dilution air and a second with cold dilution air, to lower the sample temperature. An evaporation tube is inserted between these dilution stages to bring any already condensed volatile components back to the gas phase. The $23 \mathrm{~nm}$ cut-off size was set in order to avoid measurement uncertainties that mainly originate from the inadequacy of exhaust conditioning systems (VPR), at smaller particle sizes, in delivering only solid exhaust particles without high particle losses due to diffusion or the creation of artifacts, i.e. particles being created in the VPR that did not exist in the exhaust [3]. An alternative to the evaporation tube for sub-23 nm solid particles measurement is the use of a catalytic stripper which, at suitable temperatures, removes practically all volatile material and may achieve a high particle penetration fraction with careful design $[4,5]$.

Modern internal combustion engines show a trend for emitting smaller particles. Recent studies with light duty vehicle engines show that up to $50 \%$ of the particles emitted, on a number basis, may be in the sub-23 nm region [6]. Specifically, for diesel vehicles it has been reported that possible solid nucleation particles generation mechanisms are the lubrication oil [7], the fuel additives [8] and DPF regeneration [9]. For G-DI vehicles, nucleation particles are part of the main size distribution of the exhaust [10]. Recent studies reported also solid particles generation in CNG and LPG engines [11].

In this work, we propose a simple setup for nucleation particle detection based on a supercritical DMA that operates at high temperature: the Advanced Half Mini DMA (SEADM S.L.) [12]. The Half Mini DMA was initially developed at Yale University by Fernadez de la Mora \& Kozlowski [13] and has been widely used in published work for the measurement of new particle formation either in the atmosphere [16] or in flame reactors [17] as it is the only DMA currently available that offers sufficient resolving power in the sub-3 $\mathrm{nm}$ size region. The Advanced version (denoted herein as "Advanced HM-DMA") offers high resolution, real-time measurements with 2 second time resolution for sizes up to $30 \mathrm{~nm}$ while the operation temperature can be up to $200^{\circ} \mathrm{C}[14,15]$. These unique properties render the Advanced HM-DMA suitable for engine/vehicle exhaust 
measurements with a single hot dilution stage or no dilution instead of using complex aerosol and conditioning systems that may change the solid nucleation particles size distribution. The proposed setup is not intended to apply for legislation purposes but may be used for real-time detection of solid nucleation particles emitted by internal combustion engines and may also give important information on their nature and generation mechanism.

In the following, we briefly present the principle of operation of the Advanced Half Mini DMA. We test the instrument's preciseness in the size region of interest $(5-30 \mathrm{~nm})$ using $\mathrm{NaCl}$ and soot particles. Moreover, we test the Advanced HM-DMA with hot aerosol flow to investigate the measurement repeatability under these conditions. Furthermore, we employ it to measure nucleation mode particles generated by a diesel engine using different fuels. Finally, we test the transient measurement mode of the Advanced HM-DMA in real driving emissions using a G-DI vehicle.

\section{Advanced Half Mini DMA description}

The design of the Half Mini DMA employed in this study originates from a Vienna type cylindrical nano-DMA designed to retain laminar sheath flow up to supercritical - i.e. high sheath flow - conditions needed for high resolution sizing of nanoparticles down to $1 \mathrm{~nm}$ [13]. The Advanced HM-DMA used here incorporates additional adaptations [12] which permit operation with lower sheath flow rates (and sample flows in the range of $1-2 \mathrm{lpm}$ ) in order to encompass the size range of interest for sub-23 nm combustion exhaust particles. For the current study, the Advanced HM-DMA was operated with a sheath flow of $15 \mathrm{Nlpm}$ of HEPA-filtered air and a sample flow rate of $1.5 \mathrm{Nlpm}$ and resolved particle size up to $27 \mathrm{~nm}$ approximately. With the use of heat-tolerant materials for insulating and semiconducting parts $[14,15]$ Advanced HM-DMA enables sizeselection of aerosols up to $200{ }^{\circ} \mathrm{C}$. In this work DMA was heated up to $140-150{ }^{\circ} \mathrm{C}$ to perform measurements of engine exhaust particles. Figure 1 presents the schematic of the Advanced HM-DMA principle of operation.

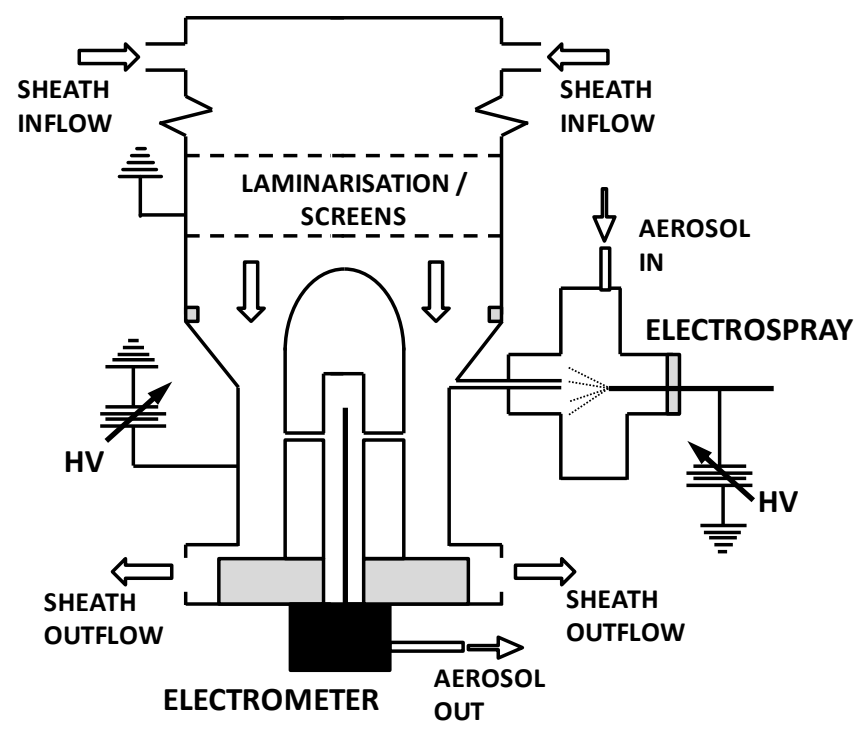

Figure 1: Schematic of the Advanced Half Mini DMA system. The DMA part is reproduced from original design by Fernadez de la Mora and Kozlowski [13] and Fernadez de la Mora [12].

Page 2 of 8
Initially, the aerosol flow is driven to a unipolar electrospray charger; the Secondary Electrospray Ionisation (SESI) charger which is adopted for charging the hot $\left(50-200{ }^{\circ} \mathrm{C}\right)$ aerosols which are of interest here. In particular, the electrospray chamber is heated at $110^{\circ} \mathrm{C}$. The Advanced HM-DMA can be also used in conjunction with a radioisotope neutraliser e.g. $\mathrm{Kr}$ (the electrospray is omitted) for cooled exhaust aerosols. The positively SESI charged particles enter the Advanced HM-DMA and are size-selected. Finally, as described in the schematic of Figure 1, the selected ionized particles are measured by means of fast electrometer with a signal amplifier of very high gain of $10^{11} \mathrm{~V} / \mathrm{A}$, developed, evaluated and coupled with DMA system by Fernadez de la Mora et al. [18].

SESI operated under identical device settings during all experiments presented in this study. The electrospray chamber was set at approximately $180 \mathrm{mbar}$, the electrospray capillary tip was always positioned at the center of chamber's inspection window while the produced current was sustained above $110 \mathrm{nA}$. We should note that the SESI charger is a novel solution - and one of the few suitable ones - for hot aerosols charging but a global charging efficiency relation has not been established due to the monopolar nature of the charger and performance dependence on variable apparatus and process conditions. Additionally, due to suspected dependence of SESI charging efficiency on the concentration of the particles being measured, we do not calculate the probability density function of the particle size distribution as it is the common practice with established particle size spectrometers. For all the above reasons, we present the Advanced HM-DMA size distributions in terms of ion concentration. Moreover, the compromise made - in order to retain the aerosol sample hot - is that the lower end of the particle size range that can be classified by the Advanced HM-DMA is now determined by the residual charged clusters or impurities from the electrospray.

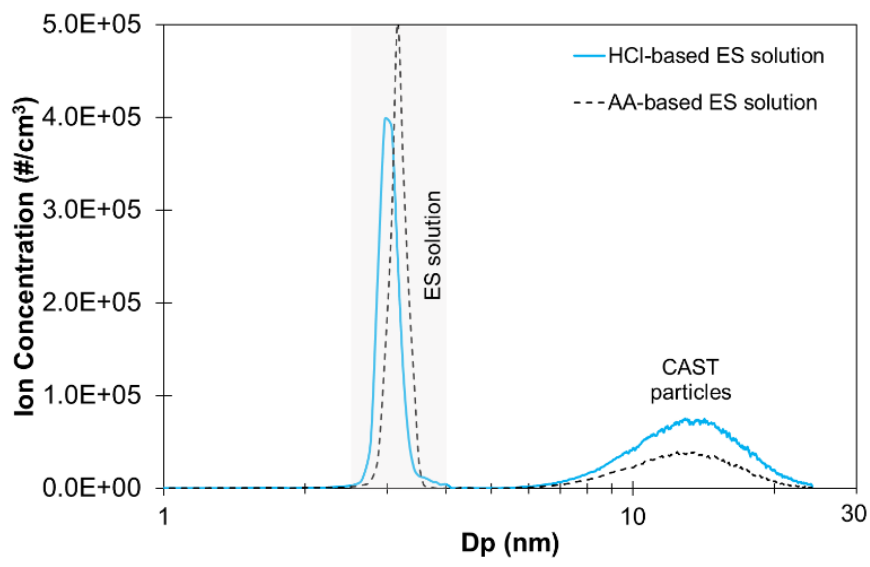

Figure 2: Comparison of Advanced HM-DMA hot operation with HCl- and ammonium acetate (AA)- based electrospray solutions, when detecting CAST sub-30nm particles. ES chamber is heated up to $110^{\circ} \mathrm{C}$, and DMA temperature is sustained at $140{ }^{\circ} \mathrm{C}$. Due to non-established charging efficiency of SESI charger the intense of signal is presented in charged particle concentration (ion concentration) instead of total particle concentration as it is the common practice.

A survey of electrospray solutions was conducted at the beginning of this work in order to determine the best composition for charging the up to $200{ }^{\circ} \mathrm{C}$ aerosol sample by the electrospray. The solutions evaluated include compositions based on DMSO/octanol or methanol, with various additions of purified water, ammonium 
acetate (AA) and/or hydrochloric acid. The selection of the electrospray solution finally adopted was based on the extent of residual "signature" created as well as the charging efficiency seen with soot aerosols from a CAST burner. For these prior studies, a CAST soot generator was run with custom settings found to produce aerosols with repeatable sub-23 nm particle modes [4]. An example comparison of hot soot aerosol charging performance for the two most prominent candidate electrospray solutions can be seen in Figure 2. The electrospray solution finally adopted is a mixture of deionised water and hydrochloric acid in DMSO/octanol, which was found to have a residual "signature" that remained at slightly smaller mobility diameter $(<5 \mathrm{~nm})$ while also exhibiting higher charging efficiency. The evaluation of the electrospray solutions was performed with the hot operation mode of Advanced HM-DMA since most of the measurements discussed in this work were also performed under this mode.

\section{Evaluation of the Advanced HM-DMA's hot operation mode}

\section{Measurement of reference aerosols}

The Advanced Half Mini DMA hot operation performance was tested with solid and thermally stable nucleation particles against reference instrumentation. For this purpose, we used an Electrospray Aerosol Generator (EAG model 3482, TSI Inc.) and a CAST generator, for $\mathrm{NaCl}$ and soot particles generation, respectively. EAG generates nanoparticles from the liquid phase with electrohydrodynamic atomization [20]. Accordingly, an aqueous solution of $\mathrm{NaCl}$ is delivered to the EAG via a syringe pump. The liquid is pushed through a capillary tube where the application of an electric field by a negatively charged orifice plate leads to the creation of highly charged droplets. Ideally, the electric field propels the liquid sample into a Taylor cone. Subsequently, the uniformly charged particles are dried by mixing with air and $\mathrm{CO}_{2}$. Finally, the nanoparticles that result from the evaporated droplets are neutralized with a soft X-ray neutralizer.

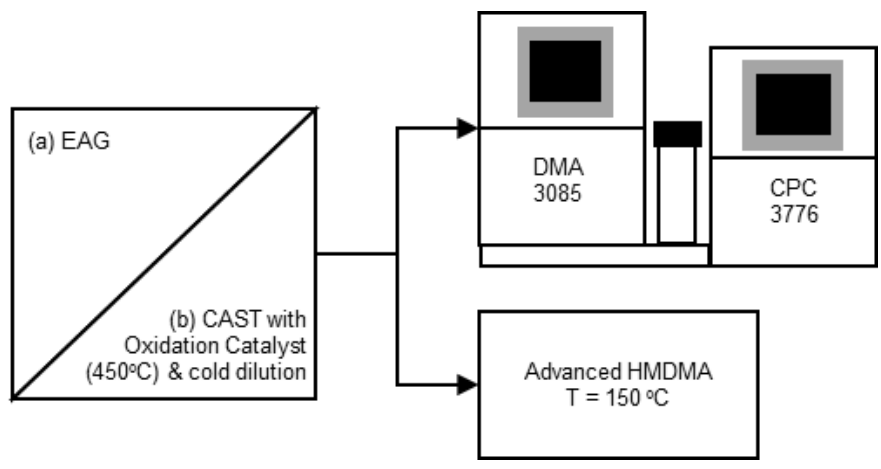

Figure 3: Experimental setup for testing the Half Mini DMA's hot operation with soot and $\mathrm{NaCl}$ particles produced by an EAG and a CAST generator.

CAST generates soot particles with a co-flow diffusion flame of propane $\left(\mathrm{C}_{3} \mathrm{H}_{8}\right)$ where the combustion air is coaxially supplied. Subsequently, soot particles are mixed with a quenching nitrogen flow to stabilize them and prevent any further combustion processes. Typical particle sizes generated by CAST lie in the 30-200 $\mathrm{nm}$ range. In non-standard operating modes, particle size may be further reduced by mixing propane with nitrogen but the volatile and semi-

Page 3 of 8 volatile fraction increases [21] and CAST-generated particles may be either volatile or solid immature soot particles with condensed organic species [22]. Thus, the generation of solid and thermally stable soot particles with CAST necessitates the addition of a volatile removal setup, preferably using a catalytic stripper (CS) instead of an evaporation tube that has been known to create artifacts [4] under some conditions. Herein, the CAST generator was followed by volatile removal setup, based on an oxidation catalyst that was maintained at $450{ }^{\circ} \mathrm{C}$. Downstream of the oxidation catalyst an ejector diluter (DEKATI, DI-1000) cools the aerosol flow to ambient temperature to permit concurrent comparison measurements. The particle size distributions that were produced by the two aerosol generators was measured with two different setups: the Advanced HM-DMA and an SMPS system. The Advanced HM-DMA was operated in its heated mode, as described in detail in section 2. The SMPS system used consisted of a nano-DMA (model 3085, TSI Inc.) and a CPC (model 3776, TSI Inc.) with cut-off size $\mathrm{d}_{50}=2.5 \mathrm{~nm}$. Figure 3 shows a schematic of the experimental setup.

In Figure 4 the particle size distribution produced by the EAG (Figure 4a) and by the CAST (Figure 4b) are shown, as measured by the Advanced HM-DMA and the SMPS. The signal of the two instruments is normalized as the charging efficiency of the Advanced HM-DMA is unknown.
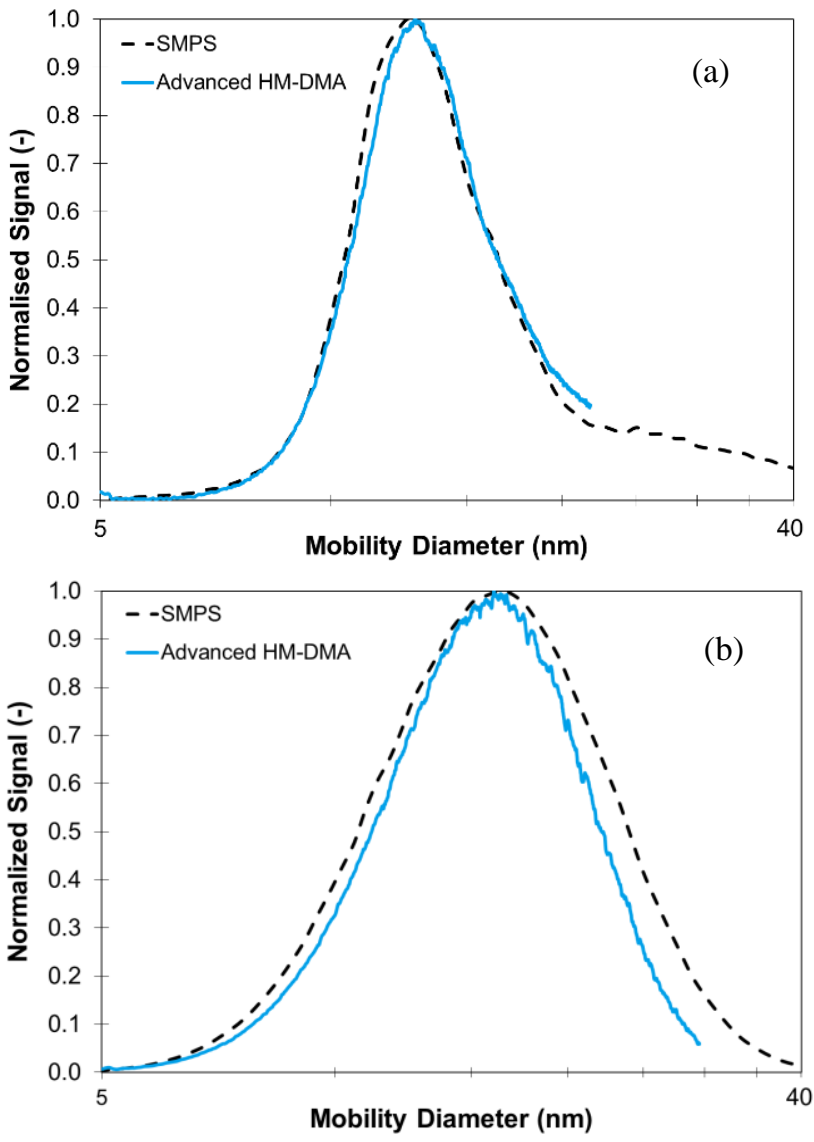

Figure 4: Normalized particle size distribution of (a) $\mathrm{NaCl}$ particles and (b) CAST-generated solid soot particles, measured with Advanced Half Mini DMA at the hot temperature operation mode and compared with an SMPS 
The normalized particle size distributions from the two instruments show an excellent agreement suggesting that the hot operation of the Advanced HM-DMA at an extended size range is highly accurate. Specifically, the mean mobility diameter, $\mathrm{d}_{\text {mean }}$, and the standard deviation, $\sigma_{\mathrm{g}}$, of the size distributions are almost identical, with the maximum difference being 5\%. Table 1 presents the mean particle diameter and standard deviation found by each instrument after 2-3 repetitions of the measurements.

Table 1. Main parameters of the particle size distributions as detected by the Advanced Half Mini DMA and the SMPS.

\begin{tabular}{|c|c|c|c|c|}
\hline $\begin{array}{c}\text { Particle } \\
\text { generator }\end{array}$ & \multicolumn{2}{|c|}{ EAG } & \multicolumn{2}{c|}{ CAST } \\
\hline Particle type & \multicolumn{2}{|c|}{$\begin{array}{c}\text { NaCl solid particles } \\
(\mathrm{N}=3)\end{array}$} & \multicolumn{2}{c|}{$\begin{array}{r}\text { Solid soot particles } \\
(\mathrm{N}=2)\end{array}$} \\
\hline $\begin{array}{c}\text { Particle size } \\
\text { distribution }\end{array}$ & $\mathrm{d}_{\text {mean }}(\mathrm{nm})$ & $\sigma_{\mathrm{g}}(-)$ & $\mathrm{d}_{\text {mean }}(\mathrm{nm})$ & $\sigma_{\mathrm{g}}(-)$ \\
\hline $\begin{array}{c}\text { Advanced } \\
\text { HM-DMA }\end{array}$ & $13.1 \pm 1.5 \%$ & $1.24 \pm 1 \%$ & $15.9 \pm 0.2 \%$ & $1.33 \pm 0.5 \%$ \\
\hline SMPS & $13.2 \pm 1.2 \%$ & $1.28 \pm 0.5 \%$ & $16 \pm 0.5 \%$ & $1.41 \pm 0.5 \%$ \\
\hline
\end{tabular}

\section{Measurement of hot aerosol particles}

Additional testing of the Advanced Half Mini DMA hot measurement accuracy was performed with hot aerosol sample. Accordingly, the CAST generator was employed to produce soot particles while an oxidation catalyst removed volatile material as previously described. Afterwards, the thermally stable solid soot particles were driven to an ejector diluter (DEKATI, DI-1000) that operated either at temperature near that of the PMP first dilution stage, i.e $150{ }^{\circ} \mathrm{C}$ or at ambient temperature, i.e. $34{ }^{\circ} \mathrm{C}$. Finally, the particle size distribution was measured by the Advanced HM-DMA within the setup shown in Figure 5.

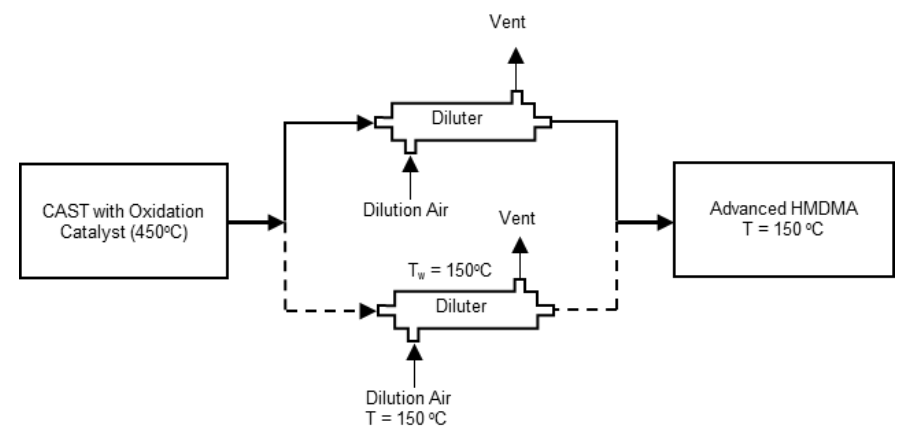

Figure 5: Experimental setup used for detecting solid particles with the prototype Advanced Half Mini DMA operating at hot mode. The aerosol sample temperature was either $150{ }^{\circ} \mathrm{C}$ or ambient.

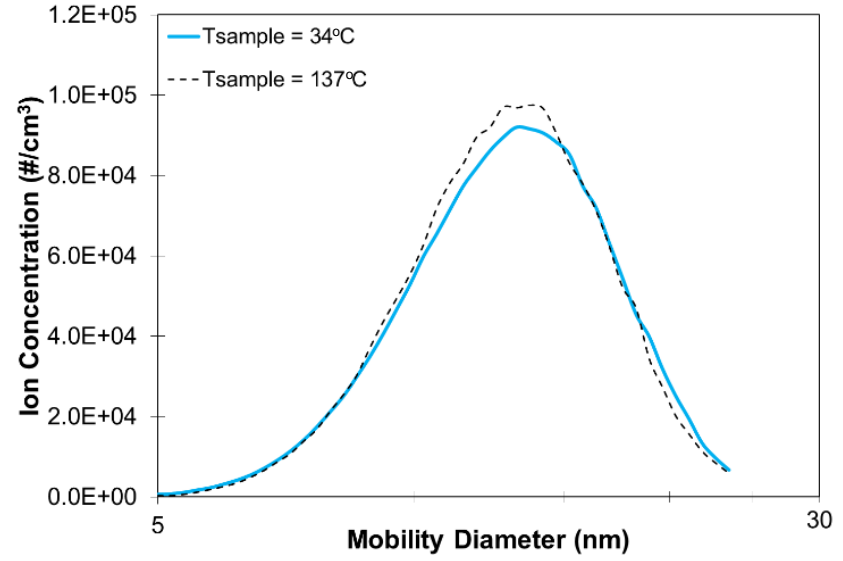

Figure 6: Advanced Half Mini DMA particle size distribution of CAST solid soot particles. Aerosol sample is either hot $\left(137^{\circ} \mathrm{C}\right)$ or close to ambient temperature $\left(34^{\circ} \mathrm{C}\right)$.

Figure 6 plots the particle size distribution that Advanced HM-DMA detects in both cases of dilution temperature. It is observed that detected mobility size distribution is identical for ambient and hot inlet aerosol flow, both in terms of size distribution characteristics $\mathrm{d}_{\mathrm{m}}=13.2 \mathrm{~nm}$ and $\sigma_{\mathrm{g}}=1.3$, as well as in terms of total ion concentration ( $<2 \%$ differences). Thus, the Advanced HM-DMA manages to measure hot sample aerosol without introducing errors.

\section{Diesel engine exhaust measurements}

\section{PMP vs single hot dilution}

The Advanced Half Mini DMA was employed to measure nucleation mode particles generated by a single cylinder, four-stroke, $5 \mathrm{~kW}$, aircooled direct injection diesel engine. We used three different fuels that result in the production of solid nucleation particles. Fuel A was a mixture of a high-sulfur fuel (1000 ppm) with a commercial lubrication oil "LiquiMoly" (SOLVAY) at a concentration of $30 \mathrm{ml}$ of lubrication oil/l of fuel. For fuels B and $\mathrm{C}$ we used a low sulfur diesel mixed either with $60 \mathrm{ml}$ of the aforementioned lubrication oil/1 of fuel (Fuel B) or with a commercial Ce-based soot oxidation catalyst by ENVIROX ("DPF Assist") in concentration of $29.4 \mathrm{ml} / 1$ fuel (Fuel C). The engine load was $23 \%$ of the maximum. Under these conditions, the diesel engine generated a bimodal distribution including nucleation particles. Measurements with the Advanced HM-DMA for Fuel A were performed either downstream a PMPcompliant sampling and conditioning system based on a catalytic stripper [4], or with a single hot dilution stage performed with an ejector diluter. For fuels $\mathrm{B}$ and $\mathrm{C}$ we measured only downstream a hot dilution stage. The simple hot dilution stage setup takes advantage of the hot Half Mini DMA operation that keeps the aerosol flow at elevated temperature avoiding any volatile particle growth or re-nucleation. Moreover, by not using a conditioning system we avoid particle losses due to diffusion which can be very substantial at small particle sizes. Figure 7 shows the experimental setup used for diesel engine measurements. 


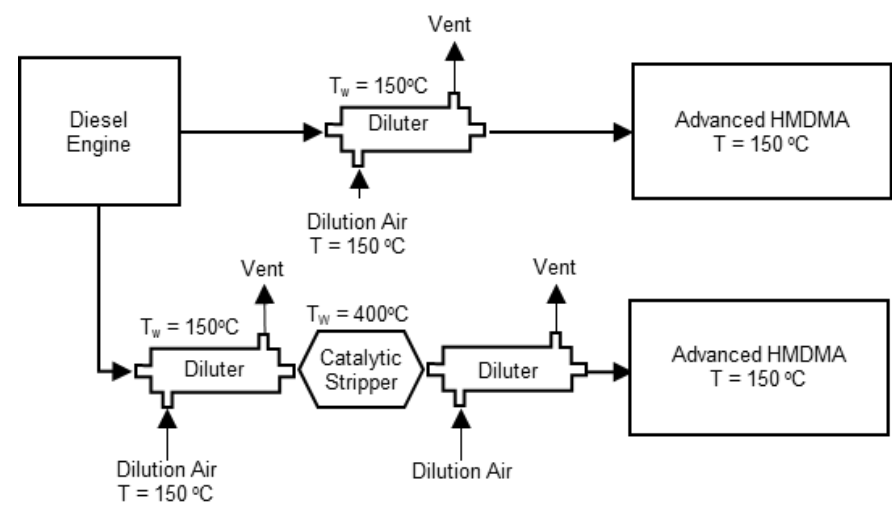

Figure 7: Experimental setup for detecting diesel solid aggregates with Advanced Half Mini DMA operating at hot mode, either sampling from onestage, hot diluter, or from a CS-based, PMP-compliant dilution system.

Figure 8 plots the particle size distributions produced by the diesel engine with Fuel A and measured by the Advanced HM-DMA with the two conditioning approaches previously described. The agreement between the measurements with the PMP-compliant and the $\mathrm{DR}=10$ hot dilution setup confirms the reliability of the Advanced HM-DMA hot operation mode and indicates the possibility of using the greatly simplified setup for solid particles measurement. We note a slight shift of the size distribution to larger particle sizes with the PMP-compliant dilution system which may be attributed to agglomeration that occurs after the hot dilution stage given that the total number concentration between the two dilution stages is $>10^{7}$ $\# / \mathrm{cm}^{3}[\mathbf{2 3}]$.

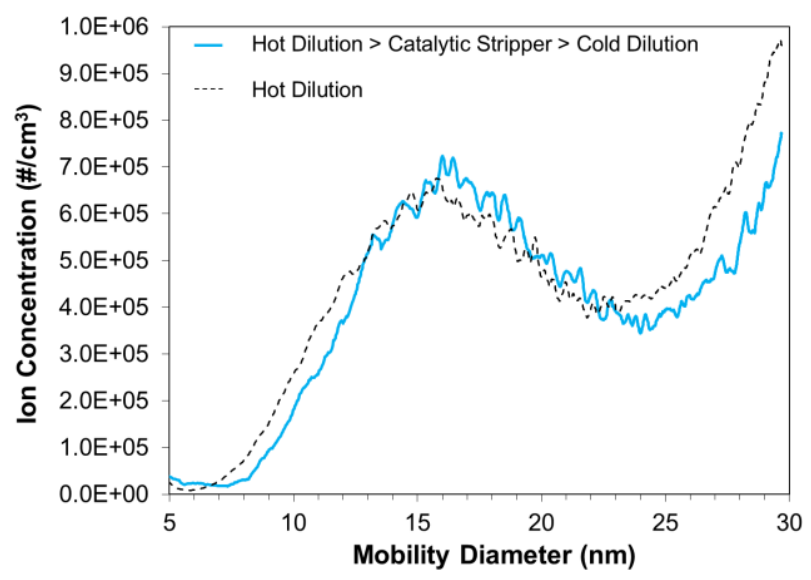

Figure 8: Sub-30nm ionised particles size distribution measured with the Advanced Half Mini DMA in hot operation using (a) a PMP-compliant setup and (b) a single step hot dilution.

Figure 9 depicts the particle size distributions produced by the diesel engine using fuels A and B and measured by the Advanced HMDMA using a single hot dilution stage. The addition of Ce-based additive and lubrication oil results in the generation of solid nucleation particle modes with $\mathrm{d}_{\text {mean }} \approx 14 \mathrm{~nm}$ and $\approx 16 \mathrm{~nm}$, respectively in each case. Again, it is demonstrated that the hot operating Advanced HM-DMA coupled with the proposed sampling approach (of minimum requirements) is capable of characterizing the exhaust solid nucleation mode with high resolution.

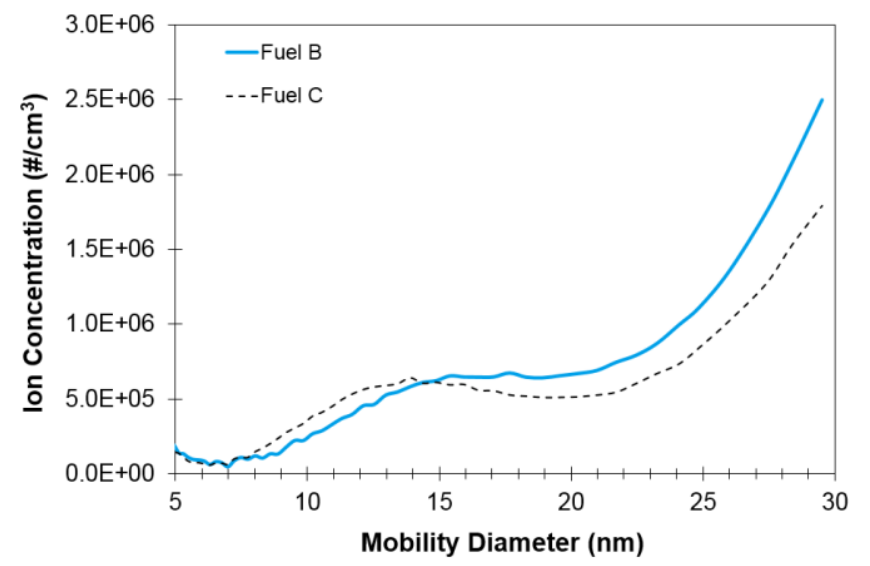

Figure 9: Sub-30nm ionized particles size distribution measured with the Advanced Half Mini DMA in hot operation. Nucleation particle mode is generated due to the addition of (a) Ce-based fuel additives and (b) lubrication oil.

\section{Transient measurements}

A portable version of the Advanced Half Mini DMA was modified appropriately in order to perform continuous real time measurements. Modifications of sweep parameters and data acquisition were proposed by the authors and realized by SEADM S.L. and allowed spectrum acquisition every 2 seconds, with a size-step of less than 1 $\mathrm{nm}$. The proof of the system's capability on performing real-time measurements was demonstrated during on-road measurements on a light-duty, Euro $6 \mathrm{~b}$ vehicle equipped with a $1000 \mathrm{cc}$, 3-cylinder, turbocharged, gasoline direct injection engine with TWC, without GPF. The vehicle exhaust was sampled directly from the tailpipe with a sampling line heated at $160^{\circ} \mathrm{C}$. The sampling port was designed and constructed according to JRC PEMS guidelines [24]. In order to avoid condensation and particles losses, the sampling tubes were thermally insulated.

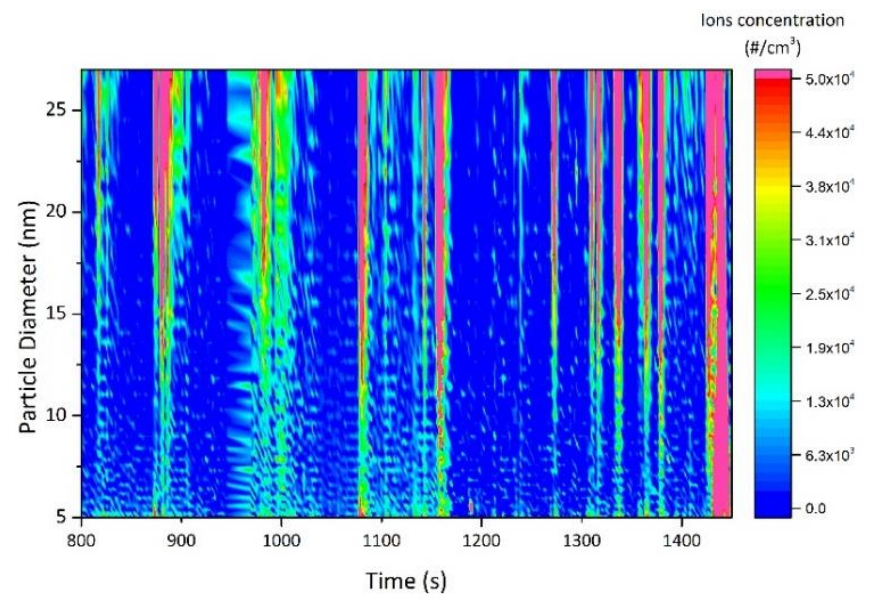

Figure 10: Real time on-road measurement using the Advanced Half Mini DMA without any dilution. 
Figure 10 shows an example of real-time measurement during a random on-road trip in a suburban area. High emissions of particulates in the range of 5-27 nm were observed during intervals of aggressive accelerations and uphill driving.

\section{Conclusions}

The Advanced Half Mini DMA is an adaptation of a supercritical DMA, initially developed for high resolution measurement of particles in the size range $1-15 \mathrm{~nm}$, at ambient temperature [13]. After recent modifications [12,14,15] the system has been shown to be capable of exhaust aerosol measurements with an extended particle size range up to $30 \mathrm{~nm}$, at high-temperature operation up to $200{ }^{\circ} \mathrm{C}$ and with real-time scanning ability $(5-30 \mathrm{~nm}$ scan every 2 seconds).

In this work we employed, for the first time, the Advanced HMDMA for measuring nucleation mode particles emitted by internal combustion engines. Initially, we tested the accuracy of the extended measurement size range with $\mathrm{NaCl}$ and soot reference aerosols and found excellent agreement in terms of size classification in the sub$30 \mathrm{~nm}$ size range comparing to an SMPS system. Additional measurements of soot solid particles were performed with both hot and ambient temperature aerosol samples demonstrating good agreement between the two measurement conditions. It is also, shown that the system resolution does not deteriorate in the case of hot operation and consequently the extended functionality may be used for exhaust measurements without introducing additional errors.

The comparison between the Advanced HM-DMA and other commercial devices is limited to size classification due to the not yet measured charging efficiency of the Secondary Electrospray Ionisation adopted for hot aerosol sample charging. Therefore, future work related to the Advanced HM-DMA will focus on understanding and determining the charging efficiency of such a unipolar charger. This is expected to be a challenging task due to the multiple dependencies of the electrospray operation and its interaction with the aerosol sample. However, establishing the charging efficiency of the SESI is necessary to fully exploit the new instrument advantages that this work demonstrates.

After the accuracy testing with reference aerosols the Advanced HMDMA was used for measuring nucleation particles produced by a diesel engine in laboratory measurements and by a G-DI vehicle in real driving conditions. Diesel engine stationary measurements were performed with a single hot dilution stage and compared to a PMPcompliant setup. The excellent agreement demonstrated in size classification between PMP compliant and single stage hot dilution setups confirms the reliability of the Advanced HM-DMA hot operation mode and indicates the possibility of using a greatly simplified setup for solid exhaust particle measurements. This approach is practically advantageous and in line with recent studies that examine the possibility of vehicle emissions tailpipe measurements [6]. The Advanced HM-DMA hot operation prevents the creation of volatile artifacts that are possible when the exhaust sample is cooled, avoids much of the particle losses inherent in complex aerosol conditioning systems and offers high resolution measurements over the size range $5-30 \mathrm{~nm}$.

\section{References}

1. Alfoldy, B., Giechaskiel, B., Hofmann, W., and Drossinos, Y., "Size-distribution dependent lung deposition of diesel exhaust particles", J. of Aerosol Science 40(8):652-663, 2009, doi:10.1016/i.jaerosci.2009.04.009.

2. Martini, G., Giechaskiel, B., and Dilara, P., "Future European emission standards for vehicles: The importance of the UN-ECE Particle Measurement Programme," Biomarkers 14, SUPPL.1:29-33, 2009, doi:10.1080/13547500902965393.

3. Swanson, .J, Kittelson, D., "Evaluation of thermal denuder and catalytic stripper methods for solid particle measurements", J. of Aerosol Science 41(12): 1113-1122, 2010, doi:10.1016/j.jaerosci.2010.09.003

4. $\quad$ Melas, A.D., Koidi, V., Deloglou, D., Daskalos, E., Zarvalis, D., Papaioannou, E., and Konstandopoulos, A.G., "Development and evaluation of a catalytic stripper for the measurement of solid ultrafine particles emissions from internal combustion engines." submitted to Aerosol Sci. Technol..

5. $\quad$ Chasapidis, L., Melas, A.D., Tsakis, A., Zarvalis, D., Konstandopoulos, A.G. "A sampling and conditioning particle system for solid particle measurements down to $10 \mathrm{~nm}$ ", submitted to $S A E$ 19ICENA.

6. Giechaskiel, B., Lähde, T., and Drossinos, Y., "Regulating particle number measurements from the tailpipe of light-duty vehicles: The next step?," Environ. Res. 172:1-9, 2019, doi: 10.1016/j.envres.2019.02.006.

7. Giechaskiel, B., Manfredi, U., Martini, G., "Engine Exhaust Solid Sub-23 nm Particles : I. Literature Survey" SAE Int. J. Fuels Lubr. 7(3):2014, doi:10.4271/2014-01-2834.

8. Kostoglou, M., Konstandopoulos, A.G., and Burtscher, H., "Size distribution dynamics of fuel-borne catalytic ceria nanoparticles" J. Aerosol Sci. 38(6):604-611, 2007, doi:10.1016/i.jaerosci.2007.04.003.

9. Kostoglou, M. \& Konstandopoulos, A.G. "Oxidative Fragmentation and Coagulation of Diesel Soot Aggregates", presented at European Aerosol Conf. 2003, EAC-2003-S1061, Aug.Sept. 2003.

10. Giechaskiel, B., Dilara, P., \& Andersson, J., "Particle Measurement Programme (PMP) Light-Duty Inter-Laboratory Exercise : Repeatability and Reproducibility of the Particle Number Method" Aerosol Sci. Technol. 42(7):528-543, 2008, doi:10.1080/02786820802220241.

11. Alanen, J., Saukko, E., Lehtoranta, K., Murtonen, T., Timonen, H., Hillamo, R., Karjalainen, P., Kuuluvainen, H., Harra, J., Keskinen, J., Rönkkö, T., "The formation and physical properties of the particle emissions from a natural gas engine", Fuel 162:155-161, 2005, doi:10.1016/j.fuel.2015.09.003.

12. Fernadez de la Mora, J., "Expanded flow rate range of high-resolution nanoDMAs via improved sample flow injection at the aerosol inlet slit", J. of Aerosol Science 11:265-275, 2017, doi:10.1016/j.jaerosci.2017.07.020.

13. Fernadez de la Mora, J., Kozlowski, J., "Hand-held differential mobility analyzers of high resolution for 1-30 nm particles : Design and fabrication considerations", J. of Aerosol Science 57:45-53, 2013, doi:10.1016/j.jaerosci.2012.10.009.

14. Amo, M., Barrios, C., del Castillo, J.C., Fernadez de la Mora, J., Konstandopoulos, A.G., Baltzopoulou, P., Vlachos, N.D., 
"Half-Mini DMA Modification for High Temperature Aerosols and Evaluation on Various Combustion Exhausts" presented at European Aerosol Conf. 2017, T311N2e5, Aug.-Sept. 2017.

15. Amo, M., Barrios, C., Delgado, R., Fernadez de la Mora, J., "A DMA operating at $200^{\circ} \mathrm{C}$ for the analysis of engine exhaust nanoparticles", presented at Aerosol Technology Conf. TA3-02, Jun. 2018

16. Cai, R., Attoui, M., Jiang, J., Korhonen, F., Hao, J., Petäjä, T., \& Kangasluoma, J., "Characterization of a high-resolution supercritical differential mobility analyzer at reduced flow rates," Aerosol Sci. Technol., 52(11):1332-1343, 2018, doi:10.1080/02786826.2018.1520964.

17. Wang, Y., Fang, J., Attoui, M., Chadha, T.S., Wang, W.N., and Biswas, P., "Application of Half Mini DMA for sub 2nm particle size distribution measurement in an electrospray and a flame aerosol reactor," J. Aerosol Sci. 71:52-64, 2014,

doi:10.1016/j.jaerosci.2014.01.007.

18. Fernadez de la Mora J., Perez-Lorenzo, L.J., Arranz, G., Amo-Gonzalez, M. \& Burtscher, H., "Fast high-resolution nanoDMA measurements with a $25 \mathrm{~ms}$ response time electrometer", Aerosol Sci. Technol. 51(6):724-734, 2017, doi:10.1080/02786826.2017.1296928

19. Daskalos, E., Melas,A.D., Deloglou, D., Zygogianni, A., Papaioannou, E., Konstandopoulos, A.G., "Assessment of a reference aerosol generated by a propane diffusion flame (CAST) in the sub-23 $\mathrm{nm}$ range", poster presented at European Aerosol Conference (EAC 2017), T311N3ff, Aug.-Sept., 2017, doi: 10.5281/zenodo.1204924

$20 . \quad$ Biskos, G., Vons, V., Yurteri, C. U., and Schmidt-Ott, A., "Generation and sizing of particles for aerosol-based nanotechnology," KONA Powder Part. J., 26,13-35, 2008, doi:10.14356/kona.26.2008006

21. Mamakos, A., Khalek, I., Giannelli R., and Spears, M., "Characterization of Combustion Aerosol Produced by a Mini-CAST and Treated in a Catalytic Stripper", Aerosol Sci. Technol. 47(8):927936, 2013, doi:10.1080/02786826.2013.802762

22. Maricq, M.M, "Examining the Relationship Between Black Carbon and Soot in Flames and Engine Exhaust," Aerosol Sci. Technol. 48(6):620-629, 2014, doi:10.1080/02786826.2014.904961

23. Giechaskiel B., Arndt M., Schindler W., Bergmann A., et al., "Sampling of Non-Volatile Vehicle Exhaust Particles: A Simplified Guide" SAE Int. J. Engines 5(2):2012, doi:10.4271/2012$\underline{01-0443}$

24. Valverde Morales V., Bonnel P., "JRC Technical reports: On-road testing with Portable Emissions Measurement Systems (PEMS) - Guidance note for light-duty vehicles", Joint Research Center, 2018, doi:10.2760/08294

\section{Contact Information}

Penelope Baltzopoulou

Centre for Research and Technology-Hellas (CERTH)

Chemical Process and Energy Resources Institute (CPERI)

Aerosol \& Particle Technology Laboratory (APTL)

$6^{\text {th }} \mathrm{km}$ Harilaou-Thermi Rd, Thessaloniki, Greece, 57001

e-mail: pbaltzop@cperi.certh.gr

\section{Acknowledgments}

The authors would like to acknowledge the European Commission, EU Horizon 2020 research and innovation program for funding the SUREAL-23 project (GA 724136) in the frame of which this work performed. The authors would also like to specially thank Prof. Juan Fernadez de la Mora (Yale University) and SEADM S.L. for the loan of Advanced Half Mini DMA (initial and modified portable version) and their continuous and valuable guidance on system operation and electrospray solutions preparation that they provided within the SUREAL-23 project.

\section{Definitions/Abbreviations}

$\mathbf{A A}$

CAST Burner

CPC

CS

CNG

CVS

DMA

DMSO

DPF

EAG

ES

GDI

GPF

HM

HM-DMA

LPG

LSD

PMP

SESI

SMPS

TWC

VPR
Ammonium Acetate

Combustion Aerosol Standard Burner

Condensation Particle Counter

Catalytic Stripper

Compressed Natural Gas

Constant Volume Sampling

Differential Mobility

Analyzer.

Dimethyl sulfoxide

Diesel Particulate Filter

Electrospray Aerosol

Generator

Electrospray

Gasoline Direct Injection

Gasoline Particle Filter

Half Mini

Half Mini Differential Mobility Analyzer (also denoted as "Advanced Half Mini DMA")

Liquefied Petroleum Gas

Low Sulfur Diesel

Particle Measurement Program

Secondary Electrospray Ionization

Scanning Mobility Particle Sizer

Three-Way Catalyst

Volatile Particle Remover 
Pre-typeset version of SAE International Technical Paper 2019-24-0052

Page 8 of 8 